\title{
Central Regions of the Early-Type Galaxies in the NGC 3169 Group
}

\author{
O. K. Sil'chenko ${ }^{1 *}$ and V. L. Afanasiev ${ }^{2}$ \\ ${ }^{1}$ Sternberg Astronomical Institute, Universitetskii pr. 13, Moscow, 119992 Russia \\ ${ }^{2}$ Special Astrophysical Observatory, Russian Academy of Sciences, \\ Nizhnii Arkhyz, 369167 Karachai-Cherkessian Republic, Russia
}

Received January 11, 2006

\begin{abstract}
We have investigated the central regions of the galaxies in the NGC 3169/NGC 3166/NGC 3156 group with the multipupil fiber spectrograph of the 6 - $m$ telescope; the first (central) galaxy in the group is a spiral ( $\mathrm{Sa}$ ) one and the other two galaxies are lenticular ones. The group is known to have an extended HI cloud with a size of more than $100 \mathrm{kpc}$ that is associated in its position, orientation, and rotation with the central galaxy NGC 3169. The mean age of the stellar populations in the centers of all three galaxies has been found to be approximately the same, $\sim 1$ Gyr. Since the galaxies are early-type ones and since NGC 3166 and NGC 3156 show no global star formation, we are dealing here with a synchronous star formation burst in the centers of all three galaxies.

PACS numbers: 98.52.-b; 98.52.Lp; 98.52.Nr; 98.62.-g; 98.62.Ai; 98.65.-r

DOI: $10.1134 / \mathrm{S} 1063773706080044$
\end{abstract}

Key words: galaxies, groups and clusters of galaxies, galactic nuclei, galactic structure, galactic evolution.

\section{INTRODUCTION}

According to the present views on cosmological evolution, the groups of galaxies are not just galaxies that happened to be side by side at a given time for some reason. The hierarchical concept of galaxy formation and evolution says that the galaxies of a group must be embedded in a common dark halo whose mass (according to theory) is an order of magnitude higher than the total mass of their stars. The common dark halo is difficult to detect in observations and the data on mass-to-light ratios in groups of galaxies are contradictory so far (Karachentsev 2005), but, at the same time, X-ray observations reveal a common hot gaseous halo in the most massive groups (Mulchaey et al. 2003). There are also several nearby groups where intergalactic neutral hydrogen is observed in the form of a regularly rotating massive disk or ring (Schneider 1985, 1989; Haynes 1981). All of these facts suggest at least a long synchronous evolution of the galaxies in groups, if not a common origin. The best conditions for the influence of the so-called external factors on the evolution of galaxies are realized in groups. These factors include the interactions with other galaxies, because, on the one hand, the galaxies are close to one another and, on the other hand, their velocities relative to the neighbors are not so high as those in clusters: the mean velocity

\footnotetext{
${ }^{*}$ E-mail: olga@sai.msu.su
}

dispersion of the galaxies in groups with an X-ray gas is only $400 \mathrm{~km} \mathrm{~s}^{-1}$ (Mahdavi et al. 2000). Therefore, it makes sense to search for evidence of synchronous galaxy evolution that can be associated with the dominance of external factors precisely in groups. Since almost all of the external mechanisms that affect the evolution of galaxies (tidal interaction, mass transfer, mergers, the pressure of a hot intergalactic medium) lead to perturbations of the global gaseous disks in galaxies and to a gas concentration in their centers, evidence of synchronous star formation bursts should be sought precisely in the galactic centers.

We already have successful experience of this kind. Recently, we have investigated the central regions of three galaxies belonging to the Leo I group, the elliptical NGC 3379, the lenticular NGC 3384, and the spiral NGC 3368, by means of two-dimensional spectroscopy (Sil'chenko et al. 2003). In this group, Schneider (1985) previously detected a giant (more than $100 \mathrm{kpc}$ in radius) HI ring that probably once interacted with all three above-mentioned galaxies. We found stellar-gaseous disks with identically oriented rotation axes in the central regions of two of the three galaxies (although the galaxies themselves are oriented differently) and these rotation axes coincide with the rotation axis of the intergalactic HI cloud. The central disk in NGC 3379 turned out to be exactly polar with respect to the other two central disks, which can be explained by the dynamical effects of 
Table 1. Global parameters of NGC 3156, NGC 3166, and NGC 3169

\begin{tabular}{l|c|c|c}
\hline \multicolumn{1}{c|}{ NGC } & 3156 & 3166 & 3169 \\
\hline Morphological type (NED) & $\mathrm{S} 0:$ & $\mathrm{SAB}(\mathrm{rs}) 0 / \mathrm{a}$ & $\mathrm{SA}(\mathrm{s}) \mathrm{a} \mathrm{pec}$ \\
Distance, Mpc (Tonry et al. 2001) & & 22.4 & 14.2 \\
$R_{25}, \mathrm{kpc}$ & 5.9 & 15.2 & 11.08 \\
$B_{T}^{0}(\mathrm{RC} 3)$ & 12.91 & 11.01 & -20.3 \\
$M_{B}(L E D A)$ & -18.5 & -20.2 & 0.78 \\
$(B-V)_{T}^{0}(\mathrm{RC} 3)$ & 0.75 & 0.87 & 0.21 \\
$(U-B)_{T}^{0}(\mathrm{RC} 3)$ & 0.26 & 0.36 & 1238 \\
$V_{r}(\mathrm{NED}), \mathrm{km} \mathrm{s}^{-1}$ & 1318 & 1345 & $54^{\circ}$ \\
Inclination (LEDA) & $79^{\circ}$ & $62^{\circ}$ & $45^{\circ}$ \\
$P A_{\text {phot }}$ & $47^{\circ}$ & $87^{\circ}$ & 165 \\
$\sigma_{*}, \mathrm{~km} \mathrm{~s}^{-1}(\mathrm{LEDA})$ & 81 & 112 & \\
\hline
\end{tabular}

gas accretion onto a rotating massive triaxial body. The age of the stellar populations in the centers of NGC 3384 and NGC 3368 is the same, 23 Gyr; this allows the quasi-synchronous central star formation burst, the source of the gas for which was probably the gas of the intergalactic ring, to be diagnosed.

The NGC 3169 group is another nearby group of galaxies with a global HI cloud. Haynes (1981) presented a map of the HI distribution in this group. The huge disk is oriented and rotates in the same way as the central galaxy of the group, but is considerably larger that it in size; the immediate neighbors of NGC 3169, the lenticular galaxy NGC 3166 and

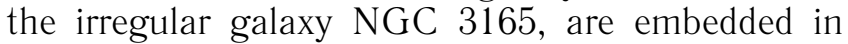
this disk (or are projected onto it?). There is another lenticular galaxy, NGC 3156, slightly farther to the southwest, outside the cloud, but on the extension of its major axis. In this paper, we investigate the kinematics and properties of the stellar populations in the centers of three galaxies, NGC 3169, NGC 3166, and NGC 3156, by means of two-dimensional spectroscopy using the multipupil fiber spectrograph of the $6-\mathrm{m}$ telescope. Table 1 lists the basic global parameters of the galaxies under study gathered from known extragalactic databases.

\section{OBSERVATIONS AND DATA REDUCTION}

The central regions of all three galaxies were observed with the multipupil fiber spectrograph (MPFS) at the prime focus of the 6-m Special Astrophysical Observatory ( $\mathrm{SAO}$ ) telescope (for a description of the instrument, see Afanasiev et al. (2001)) in the bluegreen spectral range 4200-5600 $\AA$ with a reciprocal dispersion of $0.75 \AA$ per pixel (a spectral resolution of $\sim 3.5 \AA$ ). The detector was a $2048 \times 2048$-pixel CCD array. During MPFS observations, a $16 \times 16$ microlens array builds a matrix of pupils that is fed to a grating spectrograph. Such a configuration allows 256 spectra to be taken simultaneously, each of which corresponds to a $1^{\prime \prime} .0 \times 1^{\prime \prime} .0$ spatial image element of the galaxy. We separately took the comparison spectrum of a helium-neon lamp to calibrate the wavelength scale and the dawn-sky spectrum to correct the image for vignetting and different microlens transparency. The primary data reduction (bias subtraction, cosmic ray particle hit removal, extraction of one-dimensional spectra from the CCD format, and linearization of the extracted spectra) was performed using a software package developed in the IDL programming environment by one of the authors (V.L. Afanasiev).

The MPFS observations in the blue-green spectral range were used, first, to analyze the variations in the absorption-line equivalent widths expressed as Lick indices (Worthey et al. 1994) over the field and, second, to construct the two-dimensional stellar velocity field in the galactic center. The first goal was achieved by adding the spectra in concentric rings with the center at the galactic nucleus and a radial step of $1^{\prime \prime}$, i.e., equal to the spatial element size. This allows an approximately constant signal-to-noise ratio to be maintained along the radius, which is unattainable, say, in long-slit observations; subsequently, we calculated the absorptionline indices $\mathrm{H} \beta, \mathrm{Mgb}, \mathrm{Fe} 5270$, and Fe5335. For these strong lines, there are detailed model calculations based on models for the synthesis of an old stellar population (Worthey 1994; Thomas et al. 2003). To achieve the second goal, the spectrum from each 
Table 2. Two-dimensional spectroscopic observations of the NGC 3169 group

\begin{tabular}{c|c|c|c|c|c}
\hline NGC & Date & Exposure time, min & Field of view & Spectral range, $\AA$ & Seeing \\
\hline 3156 & Apr. 12/13, 2005 & 100 & $16^{\prime \prime} \times 16^{\prime \prime}$ & $4200-5600$ & 1.8 \\
3166 & Mar. 29/30, 2003 & 45 & $16 \times 15$ & $4200-5600$ & 1.9 \\
3166 & Jan. 20/21, 1998 & 60 & $10.4 \times 15.6$ & $6200-6850$ & 2.5 \\
3169 & Apr. 11/12, 2005 & 120 & $16 \times 16$ & $4200-5600$ & 1.9 \\
3169 & Jan. 20/21, 1998 & 60 & $10.4 \times 15.6$ & $6200-6850$ & 2.5 \\
\hline
\end{tabular}

spatial element after the continuum subtraction and its transformation to the velocity scale was crosscorrelated with the spectra of $\mathrm{K} 0-\mathrm{K} 2$ giant stars observed on the same night with the same instrumentation as the galaxy. The accuracy of the wavelength scale and the zero point of the measured velocities were controlled using the night-sky $\lambda 5577 \AA$ line. We estimate the accuracy of our individual line-of-sight velocity measurements for stars as $10 \mathrm{~km} \mathrm{~s}^{-1}$ and the accuracy of our equivalent width determinations in azimuthally averaged spectra as $0.1 \AA$.

To analyze the ionized gas kinematics, we also used the spectra of NGC 3169 and NGC 3166 take in the red spectral range $6200-6800 \AA$ with an old MPFS version on January 20-21, 1998. The spectrograph was then called a multipupil field spectrograph and operated in the so-called tiger mode (Bacon et al. 1995; Afanasiev et al. 1996). The detector was then a Russian-made $520 \times 580$-pixel CCD array produced by the Elektron Research and Production Association. The field of view was $8 \times 12$, and the spatial element size was $1^{\prime \prime} .3$. The reciprocal dispersion of the spectra was $1.57 \AA$, and the spectral resolution was $\sim 3 \AA$. These spectra were reduced using a software package developed by Vlasyuk (1993). The accuracy of the ionized gas radial velocities estimated from the centroid positions of weak emission lines is $\sim 20 \mathrm{~km} \mathrm{~s}^{-1}$.

A detailed $\log$ of observations for the galaxies of the NGC 3169 group is presented in Table 2.

\section{THE STELLAR POPULATIONS IN THE CENTERS OF NGC 3156, NGC 3169, AND NGC 3166}

The so-called degeneracy of the age and metallicity effects is a challenging problem facing investigators that wish to determine the stellar population characteristics from integrated colors or from absorption lines in integrated spectra. It is well known that both young stellar populations with solar metallicity and old globular clusters with metallicity that is two orders of magnitude lower than its solar value can have identical (blue) colors (Zasov and Sil'chenko 1983). The best way out here is to compare two colors or indices with different sensitivities to age and metallicity. For example, the (magnesium or calcium) metal-line equivalent width-hydrogenline equivalent width diagram (Sil'chenko 1993; Worthey 1994) may be considered; here, the calcium or magnesium index is a metallicity indicator, while the Balmer absorption-line index is an age indicator. However, even the first attempts of this kind to compare observations with models of stellar populations synthesized from Galactic stars (Worthey 1994; Sil'chenko 1994) showed that different metal lines could yield different metallicity and age estimates for the stellar population, in particular, for elliptical galaxies. For most galaxies, the abundance ratio of different groups of chemical elements was found to differ from its solar value and the most significant difference here is a variable ratio of the abundances of $\alpha$-elements (oxygen, magnesium, etc.) to those of iron-peak elements. This effect is explained in terms of current models for chemical evolution by a different duration of the star formation epoch: $\alpha$-elements are produced mainly by type II supernovae (SNe II), while iron is produced by type Ia supernovae ( $\mathrm{SNe}$ Ia). If the star formation burst was short, the nucleosynthesis products of $\mathrm{SNe}$ Ia whose mass explosions begin $\sim 1$ Gyr after the onset of star formation will have no time to enter young stars and the $\mathrm{Mg} / \mathrm{Fe}$ ratio will be a factor of 2-3 higher than its solar value (see, e.g., Matteucci 1994). In contrast, the solar element abundance ratio is formed during long steady star formation over a period of several Gyr. At last, model stellar population synthesis calculations have also appeared most recently for a variable $\mathrm{Mg} / \mathrm{Fe}$ ratio. In this paper, we use models from Thomas et al. (2003) to determine the stellar population characteristics.

Figure 1 presents a diagram that maps the absorption-line index $\mathrm{H} \beta$ to the combined metalline index $[\mathrm{MgFe}] \equiv(\mathrm{Mg} b\langle\mathrm{Fe}\rangle)^{1 / 2}$, where $\langle\mathrm{Fe}\rangle \equiv$ $(\mathrm{Fe} 5270+\mathrm{Fe} 5335) / 2$. Together with our measurements of the azimuthally averaged indices at differ- 


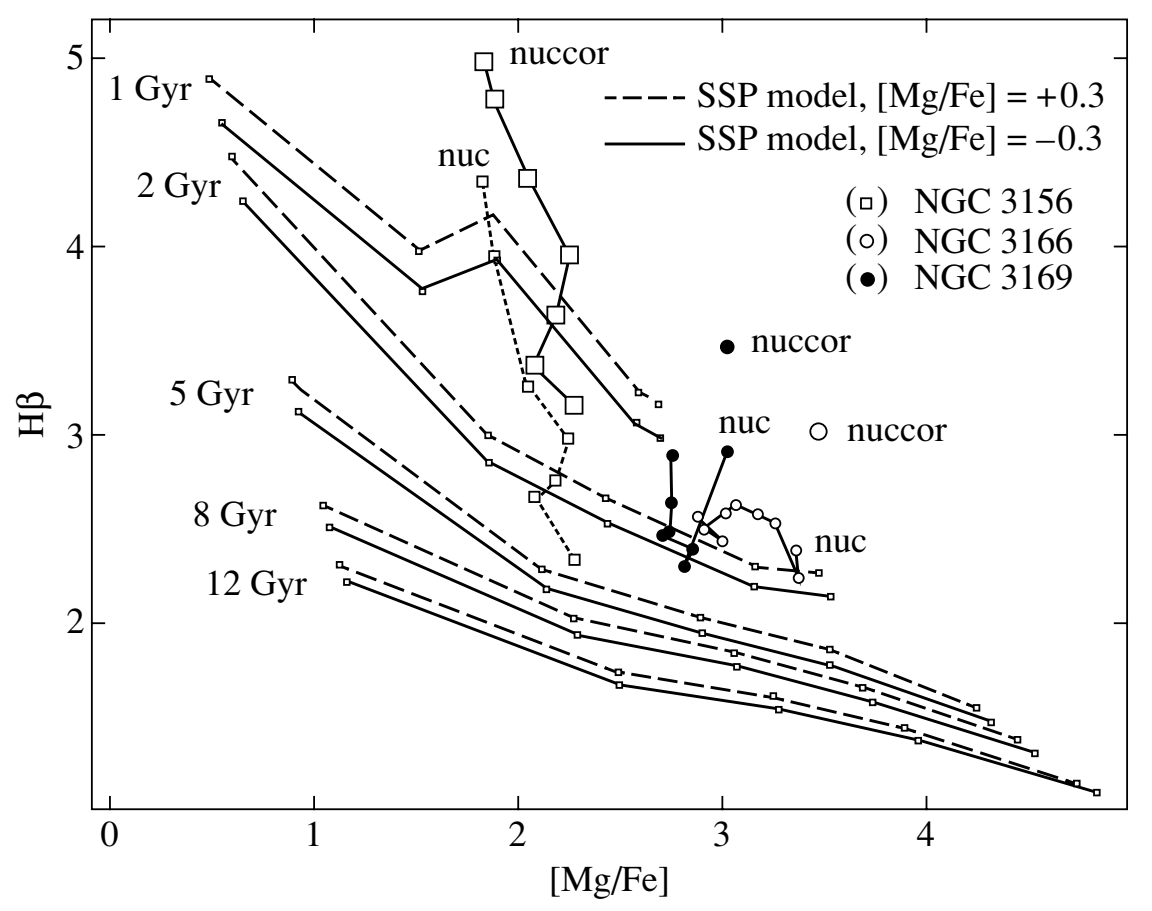

Fig. 1. Lick index diagram to diagnose the stellar population age. The line-connected symbols represent the azimuthally averaged index measurements in galaxies taken along the radius at $1^{\prime \prime}$ steps. The larger symbols represent the $\mathrm{H} \beta$ indices corrected for emission as described in the text. The data are compared with models of "simple" stellar populations (one age, one metallicity) from Thomas et al. (2003) for two values of $[\mathrm{Mg} / \mathrm{Fe}]$. The dots in the model curves of constant age, taken from right to left, mark the metallicities $[\mathrm{Z} / \mathrm{H}]+0.67,+0.35,0.00,-0.33$, and -1.35 .

ent distances from the center in the three galaxies under study, we also plotted models from Thomas et al. (2003) in this diagram for two magnesium-toiron abundance ratios, +0.3 and -0.3 . We see that if the stellar population is magnesium overabundant, then the age estimates will be slightly higher for the same values of the $\mathrm{H} \beta$ index, but, in principle, the combined metal-line index allows the acuteness of the problem to be reduced: the uncertainty in the age estimate due to the uncertainty in the $\mathrm{Mg} / \mathrm{Fe}$ ratio in this diagram is of the same order of magnitude as the uncertainty due to the statistical errors of our observations.

Before we compare our observations with stellar population models, we must solve another serious problem. The $\mathrm{H} \beta$ equivalent width, or index, can be distorted by weak emission; in early-type galaxies, this can be critical mainly for the nuclei. To correct the $\mathrm{H} \beta$ indices that we measured in the galactic nuclei for emission, we decided to use the equivalent width of the $\mathrm{H} \alpha$ emission line, since the $\mathrm{H} \alpha$ emission is always much stronger than the $\mathrm{H} \beta$ emission and since the $\mathrm{H} \alpha$ absorption line is not deeper than the $\mathrm{H} \beta$ absorption line in the integrated spectra of a stellar population of any age, while in the spectra of intermediate-age stellar populations (with which, as will be seen below, we are dealing in the case under consideration), it is much shallower than the higherorder Balmer lines (Balinskaya and Sil'chenko 1992). The emission-line ratio $\mathrm{H} \alpha / \mathrm{H} \beta$ has been studied well both theoretically and observationally; the minimum value of this ratio, $\sim 2.5$, is typical of the radiative excitation of gas by young massive stars (Burgess 1958), while this ratio is even higher for other excitation mechanisms. Since the galaxies being studied here do not belong to galaxies with H II region-type nuclei (according to the classification by Ho et al. 1997), we use the formula $E W\left(\mathrm{H} \beta_{\text {emis }}\right)=0.25 E W\left(\mathrm{H} \alpha_{\text {emis }}\right)$ for them: this mean ratio was obtained by Stasinska and Sodré (2001) for a large inhomogeneous sample of nearby emission-line galaxies. We took $E W\left(\mathrm{H} \alpha_{\text {emis }}\right)$ for the nuclei of NGC 3169 and NGC 3166 from Ho et al. (1997). Unfortunately, being too faint, NGC 3156 was not included in their sample and we have no observations in the red spectral range for this galaxy; therefore, we had to use a more traditional method of correcting the $\mathrm{H} \beta$ index for emission through the equivalent width of the [O III] $\lambda 5007$ emission line. Here, we used recommendations by Trager et al. (2000): they determined the mean ratio $\Delta \mathrm{H} \beta=0.6 E W$ ([O III] $\lambda 5007)$ from a sample of elliptical galaxies, although they pointed out that the individual $\mathrm{H} \beta /[\mathrm{O}$ III] ratios could vary within the range $0.33-1.25$. In NGC 3156, we found a strong 
[O III] $\lambda 5007$ emission line over the entire field under study; Fig. 1 shows both the original measurements and the emission-corrected measurements for the entire range of distances from the center in order that the significance of the correction for the age estimate could be estimated by eye. In the other two galaxies, the emission in the nucleus is weaker than that in NGC 3156, while at distances of several arcseconds from the center it vanishes altogether.

Let us now turn to the estimates of the (luminosityweighted) mean age of the stellar populations in the central regions of NGC 3169, NGC 3166, and NGC 3156, which can be made using the diagram in Fig. 1 and models from Thomas et al. (2003). In all three galaxies, these estimates are similar and the age itself is very young, given that we are dealing with early-type galaxies. All three nuclei show a mean age of $\sim 1$ Gyr; whereas the metallicity is nearly solar in the nucleus of the dwarf lenticular galaxy NGC 3156, it is more than a factor of 5 higher than its solar value in the other two, more massive galaxies. There is strong evidence of recent intense and synchronous star formation bursts. However, not only the galactic nuclei, but also their immediate neighborhoods are young: although the age varies differently with distance from the center in the three galaxies, it nowhere exceeds 2 Gyr. The behaviors of the mean stellar population age with distance from the center are different in all three galaxies. In NGC 3156, we see a clear gradient; the population becomes older with increasing distance from the center. In contrast, in NGC 3166, there is a metallicity gradient, but the stellar age remains approximately constant up to a distance of $\sim 8^{\prime \prime}$ from the center. In NGC 3169, the starlike nucleus is younger than its immediate neighborhoods by $\sim 1$ Gyr; however, a ring of the same young population as that in the nucleus shows up at $6^{\prime \prime}$ from the center. Below, we will attempt to relate these peculiarities in the geometry of the central star formation bursts to the structure of the galactic central regions.

Let us now look at the $\langle\mathrm{Fe}\rangle-M g b$ diagram (Fig. 2). The models by Thomas et al. (2003) were computed for several $\mathrm{Mg} / \mathrm{Fe}$ ratios and the families of curves in the $\langle\mathrm{Fe}\rangle-\mathrm{Mg} b$ diagram that represent each stellar population of different ages and metallicities for a given $\mathrm{Mg} / \mathrm{Fe}$ are well separated, which allows the $\mathrm{Mg} / \mathrm{Fe}$ ratio in the stellar population to be estimated by eye. We see that the galactic nuclei have the greatest differences in $\mathrm{Mg} / \mathrm{Fe}$ ratio: $[\mathrm{Mg} / \mathrm{Fe}] \approx+0.3$, $[\mathrm{Mg} / \mathrm{Fe}]=+0.1 \ldots+0.2$, and $[\mathrm{Mg} / \mathrm{Fe}]=-0.3$ in the nuclei of NGC 3169, NGC 3166, and NGC 3156, respectively! There is a clear correlation of the $\mathrm{Mg} / \mathrm{Fe}$ ratio in the nucleus with the luminosity (mass) of the entire galaxy: the star formation bursts are found to be very short in the nuclei of massive galaxies. However, the situation with NGC 3156 is almost unique. An iron overabundance relative to magnesium is encountered in irregular galaxies, but the $\alpha$-elements, on the contrary, are more often overabundant among early-type galaxies. Among the several tens of nearby lenticular galaxies in which we investigated the central stellar populations, only one galaxy, also the dwarf NGC 5574, has an iron overabundance relative to magnesium in the nucleus. Following the models of chemical evolution by Gilmore and Wyse (1991) and Marconi et al. (1994), we interpret this $\mathrm{Mg} / \mathrm{Fe}$ ratio as evidence for several discrete star formation bursts in the nucleus, with an interval between star formation bursts of 2-3 Gyr. In other words, the star formation burst 1 Gyr ago whose consequences we now see best in the nucleus of NGC 3156 was not the first one.

\section{THE KINEMATICS OF GAS AND STARS IN THE CENTERS OF NGC 3156, NGC 3169, AND NGC 3166}

Two-dimensional spectroscopy gives a complete set of spectroscopic data for the galactic region under study, including the velocity field constructed from absorption lines (for stars) and from emission lines (for ionized gas). There is not much ionized gas in early-type galaxies; its excitation is generally of a liner type and, hence, the strongest lines are low-excitation forbidden lines; in the optical spectral range, these include the [N II] $\lambda 6583$ line whose measurements we use for NGC 3169 and NGC 3166. For NGC 3156, we have no red spectrum, but it also exhibits a strong emission line in the green spectrum, [O III] $\lambda 5007$. We use its measurements to construct the ionized gas velocity field in this dwarf lenticular galaxy.

Figures 3-5 present the kinematic measurements for all three galaxies: the stellar velocity field, the stellar velocity dispersion field, the ionized gas velocity field, and, for the completeness of information, the emission-line map.

NGC 3156 (Fig. 3) gives an example of a wellcoordinated rotation of stars and gas in a plane that is, obviously, close to the symmetry plane of the galaxies, because the direction of the maximum apparent radial velocity gradient is close to the major axis of the galactic isophotes. The gas emission is distributed in a narrow strip that is also elongated along the major axis of the continuum isophotes, and we may suggest that we see the gas disk stably rotating in the symmetry plane of the galaxy or in the plane of the stellar disk. The stellar kinematic maps lead us to conclude that the kinematic center may be displaced relative to the photometric center by about $1^{\prime \prime}-1.5^{\prime \prime}$ to the southeast. This is not surprising, given that 


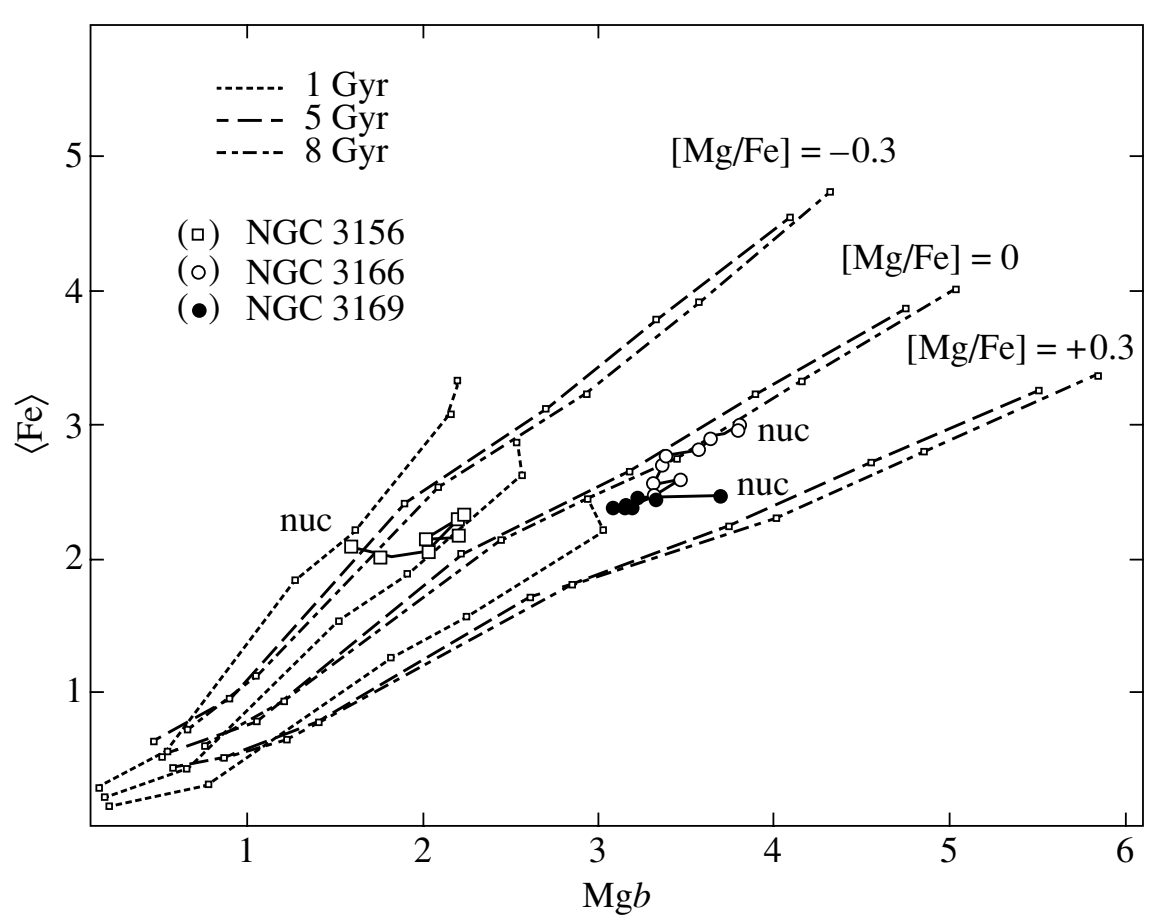

Fig. 2. Lick index diagram to diagnose the magnesium-to-iron abundance ratio in a stellar population. The line-connected symbols represent the azimuthally averaged index measurements in galaxies taken along the radius at $1^{\prime \prime}$ steps. The data are compared with models of "simple" stellar populations (one age, one metallicity) from Thomas et al. (2003) for three values of $[\mathrm{Mg} / \mathrm{Fe}]$. In each triple, the curves are given at a fixed $[\mathrm{Mg} / \mathrm{Fe}]$ ratio for three ages $-1,5$, and 8 Gyr. The dots in the model curves of constant age, taken from right to left, mark the metallicities $[\mathrm{Z} / \mathrm{H}]+0.67,+0.35,0.00,-0.33,-1.35$, and -2.25 .

there is dust at the very center of the galaxy, as implied by HST data (Tran et al. 2001). The displacement of the narrow extended stellar velocity dispersion minimum relative to the isophotal center is then also understandable: this is the circumnuclear cold stellar disk embedded in the bulge. However, the stellar velocity dispersion in the bulge is also low, only 70$80 \mathrm{~km} \mathrm{~s}^{-1}$ : as we have already mentioned, the galaxy belongs to dwarf galaxies.

In the center of NGC 3166 (Fig. 4), the picture is much more puzzling. Clearly, the stellar kinematics here is also determined by a compact circumnuclear stellar disk. The lines of equal velocities (isovels) crowd in a very distinctive way near the isophotal major axis and we even see the kinematic edge of this circumnuclear disk at $6^{\prime \prime}-7^{\prime \prime}$ from the center. The velocity dispersion also has minima near the isophotal major axis (except the nucleus itself) - this is evidence of a cold stellar subsystem. The dynamical center is also displaced relative to the photometric center, but this time along the major axis to the east. Analysis of HST images for the center of NGC 3166 in a band close to $V$ shows that the dust lanes in the nucleus of this galaxy cross the isophotal major axis at a considerable angle, almost in a polar direction, and, hence, can give the observed displacement of the dynamical center relative to the photometric center.
The gas behavior looks puzzling. The emission distribution is considerably more circular than the continuum distribution, its major axis does not coincide with that of the continuum isophotes, and the ionized gas rotation is cylindrical. We may conclude that the gas distribution and kinematics, at a fleeting glance, do not closely follow the stellar kinematics; although the gas seemingly rotates regularly, it is unlikely that the central gaseous disk in NGC 3166 may be considered well "settled" and be in total equilibrium. The twisted dust lanes in the nucleus of NGC 3166 confirm this conclusion.

The presence of a circumnuclear stellar disk in the center of NGC 3169, the most massive, central galaxy of the group (Fig. 5), is least obvious: the stellar rotation is most likely cylindrical and the stellar velocity dispersion field exhibits an extended maximum in the center. The ionized gas rotates rapidly, but its kinematic major axis turns within the MPFS field of view. The nitrogen-line emission distribution is clearly elongated in shape, and the direction of the elongation does not coincide with the direction of the kinematic major axis. The conclusion is that we see evidence of noncircular gas rotation in the center of NGC 3169; a compact bar may be present.

In Fig. 6, we compare the orientations of the photometric and kinematic major axes in the center for 

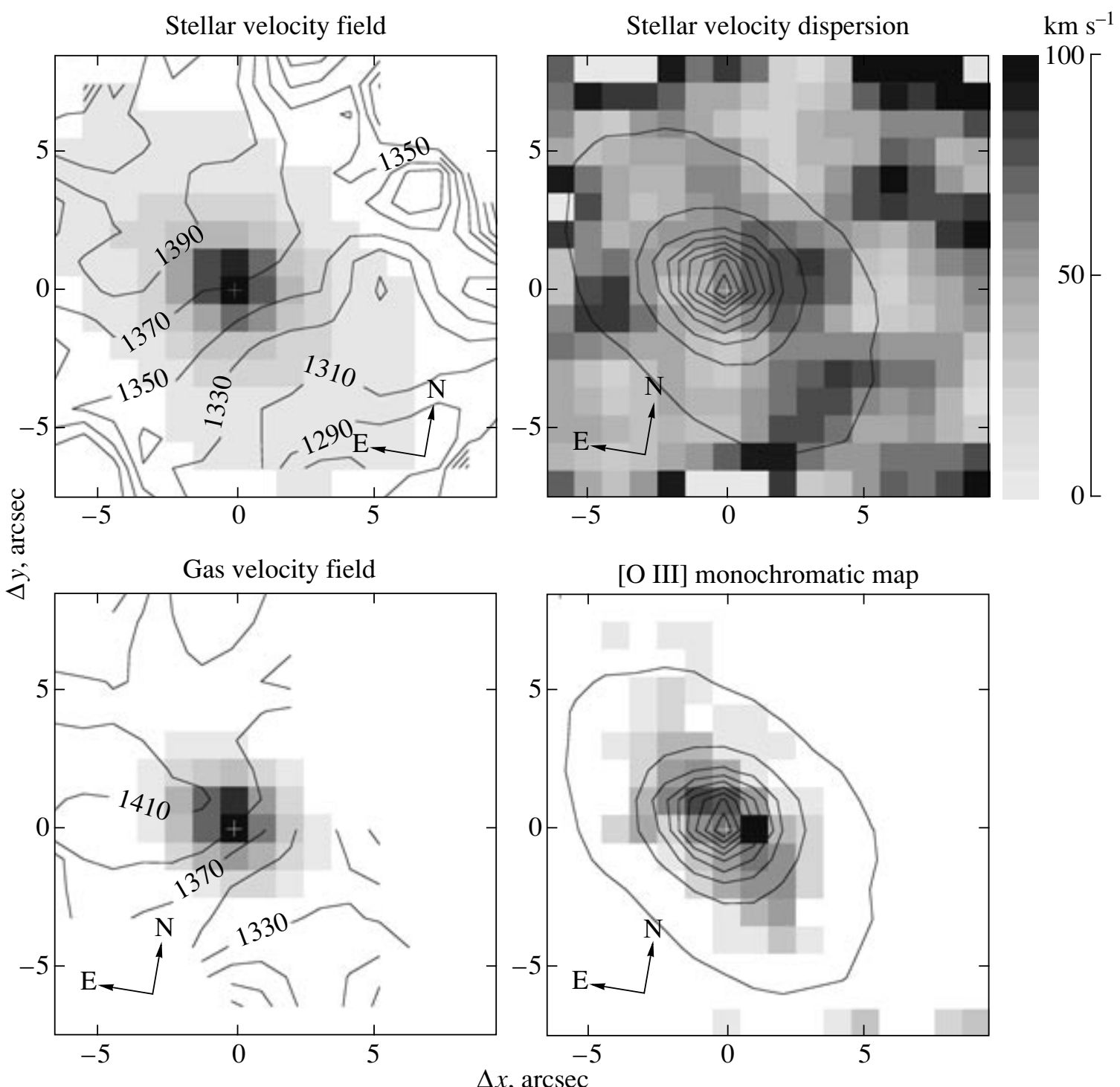

Fig. 3. Four kinematic maps for NGC 3156: on the left-the upper and lower maps present isovels of the stellar and ionized gas velocity fields, respectively, both against the background of the continuum intensity given by different shades of gray; on the right-the stellar velocity dispersion is at the top and the emission-line intensity is at the bottom, both are represented by different shades of gray; the isolines superimposed on the maps characterize the continuum intensity distribution.

each of the three galaxies. Since all three galaxies have central dust lanes, it would be natural to use infrared images to estimate the shape of the stellar density distribution. These are available for NGC 3169, the NICMOS/HST image, and NGC 3166, the image from the Ohio State University Bright Spiral Galaxy Survey (Eskridge et al. 2002); both images were obtained in a photometric band corresponding to the standard $H$ band. Since we found no deep infrared image for NGC 3156, we used its HST image with the F814W filter (which roughly corresponds to Johnson's $I$ band).

By comparing the orientations of the photometric and kinematic major axes, we can check whether the potential in which the stars and gas rotate is axisymmetric (the simplest, dynamically "cold" type of axisymmetric rotation is disk rotation). If the stellar potential is axisymmetric, the orientations of the photometric and kinematic major axes in the absence of ordered radial motions (gas flows) must coincide. Calculations for the rotation of gas and stars in the presence of a bar show that, in this case, the kinematic and photometric major axes diverge in different directions relative to the line of nodes: the lines of equal velocities tend to elongate along the bar (Moiseev and Mustsevoi 2000). What do we actually see in the galaxies under study? In NGC 3166, all orientations, the kinematic major axes of the gas and stars, 

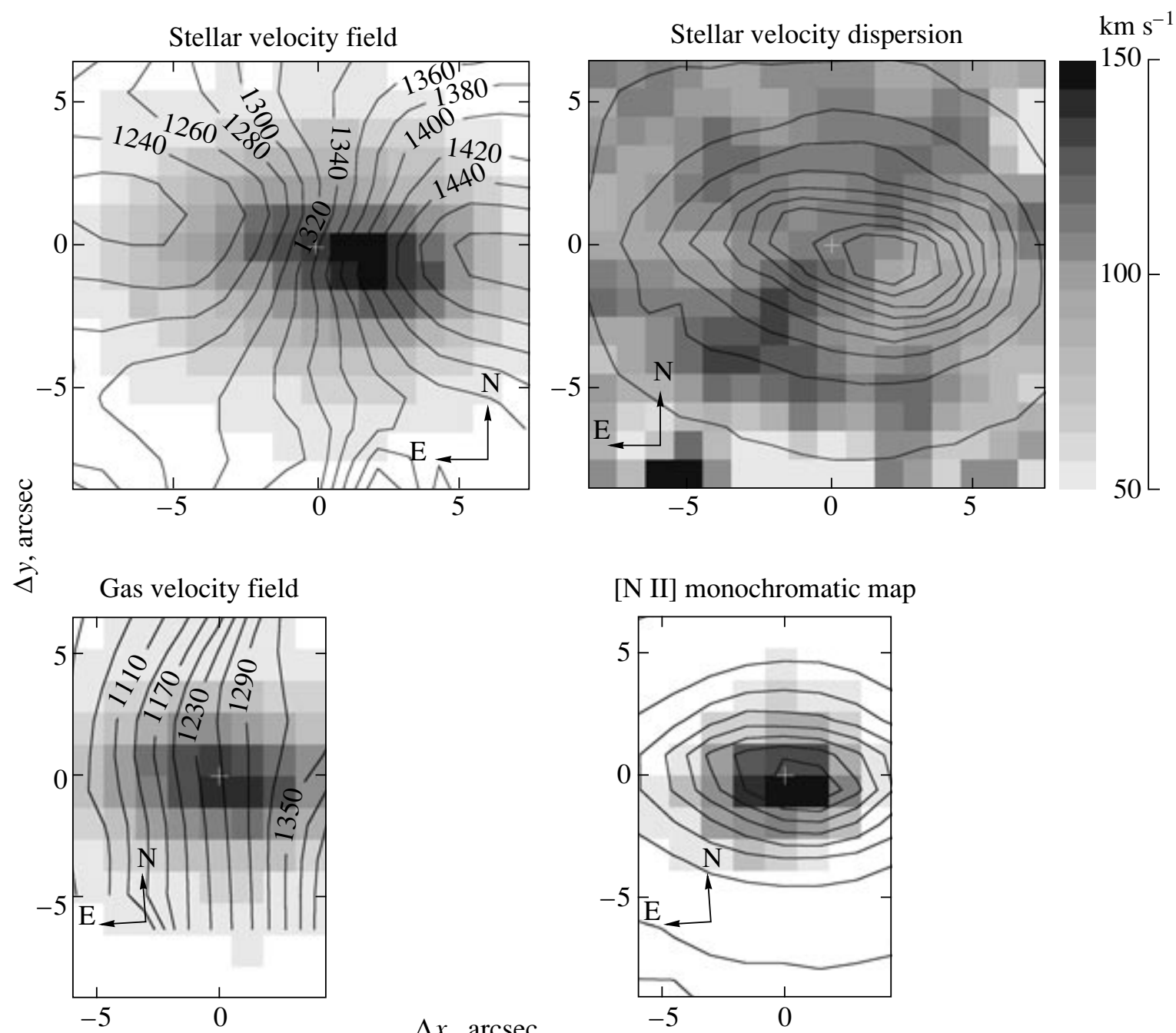

$\Delta x, \operatorname{arcsec}$

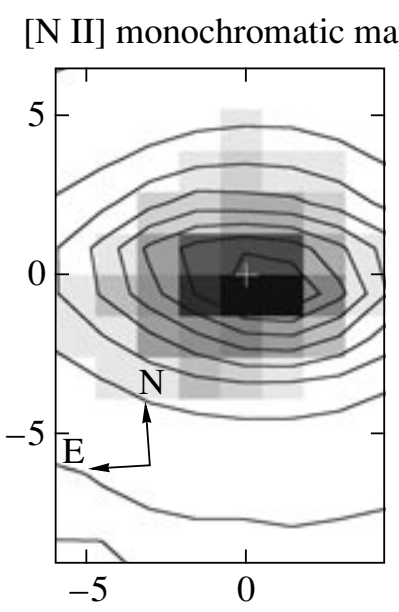

Fig. 4. Same as Fig. 3 for NGC 3166.

the photometric major axis of the central isophotes, and the line of nodes, coincide, within $\pm 3^{\circ}$; this is consistent with the conclusion that the stars and gas in the galactic center rotate axisymmetrically in the symmetry plane of the galaxy. In NGC 3156, the orientations of the kinematic and photometric major axes coincide, but both systematically and concordantly deviate from the line of nodes determined from the orientation of the isophotal major axis for the global galactic disk. This looks as if the disk plane were warped in the galactic center by $\pm 10^{\circ}$ from the global symmetry plane. In NGC 3169 , the photometric and kinematic major axes also deviate from the line of nodes by $\pm 10^{\circ}$, but they do this out of phase. The latter behavior is typical of the case where a bar is present in the stellar disk.

\section{DISCUSSION AND CONCLUSIONS}

Our analysis of the stellar population age in the centers of the three early-type galaxies in the NGC 3169 group showed that a fairly young stellar population, no older than 1-2 Gyr, dominates in them. We are dealing with the luminosity-weighted mean stellar age; it is this parameter that is determined from the integrated spectrum of the stellar system. Since the brightest and, hence, most massive stars give the largest contribution to the composite spectrum of the stellar system, stellar population synthesis shows that this weighted mean age roughly corresponds to the age of the last significant star formation burst; i.e., a synchronous star formation burst may be said to have occurred in the centers of all three galaxies of the NGC 3169 group 1 Gyr ago.

As regards lenticular galaxies, and two of the three galaxies (NGC 3166 and NGC 3156) are precisely lenticular ones, this young age of the stellar nuclei is a 

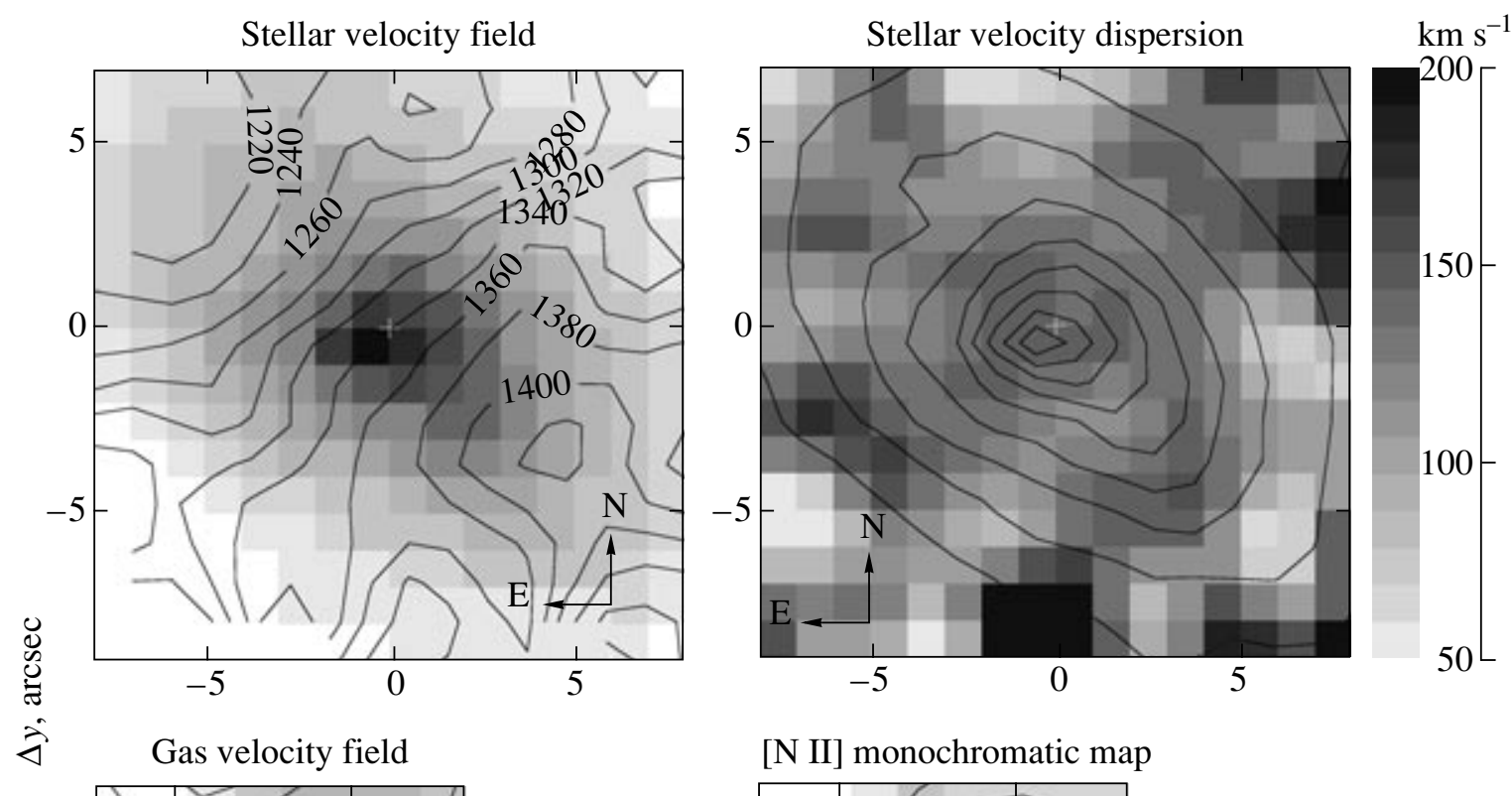

[N II] monochromatic map

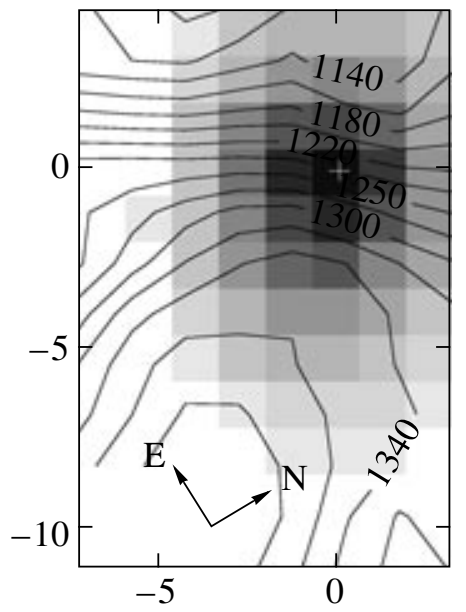

$\Delta x, \operatorname{arcsec}$

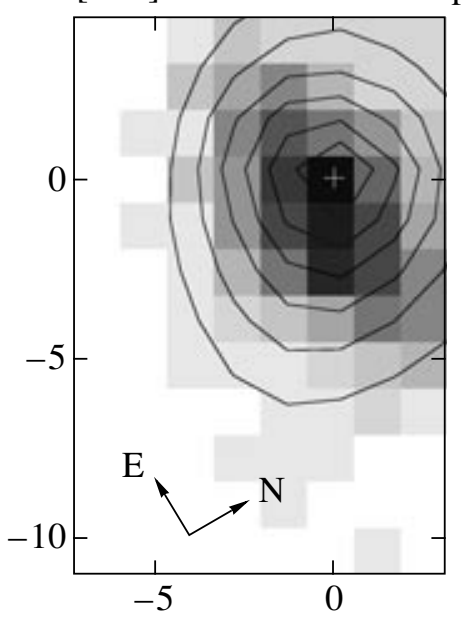

Fig. 5. Same as Fig. 3 for NGC 3169.

kind of a record: our recent statistical analysis of nearby lenticular galaxies (Sil'chenko 2006) showed that the median ages are 4-6 Gyr for the nuclei of lenticular galaxies (depending on the environment density and the position in the group) and even older, 5$8 \mathrm{Gyr}$, for the bulges. Since gas supplies from outside are required for star formation bursts in early-type galaxies that generally have no interstellar medium in sufficient amounts, it would be natural to associate the synchronous nuclear evolution of the three galaxies not with pair interactions, but with the interaction of each of them with the global HI cloud that exists in the NGC 3169 group (Haynes 1981). We reached a similar conclusion when we investigated the earlytype galaxies in the Leo I group, which also has large reserves of intergalactic neutral hydrogen (Sil'chenko et al. 2003).

The geometry of these circumnuclear star forma- tion bursts is of considerable interest. As would be expected, since the gas is dissipative in nature, it flows to the galactic center to form circumnuclear dense compact disks there and the newly forming stars, in turn, concentrate in the circumnuclear stellar disks. The large disks (the symmetry planes) of NGC 3169 and NGC 3156 may be coplanar to one another and to the intergalactic cloud, because the orientations of the major axes of the isophotes and their apparent ellipticities roughly coincide, but their central regions rotate toward one another. The systemic velocity of NGC 3156 is higher than that of NGC 3169 and, although this dwarf is already outside the intergalactic cloud, the direction of its motion in the group agrees with the direction of rotation of the intergalactic cloud as if it were entirely formed from this gas. The rotation of its circumnuclear gas can then be classified as 

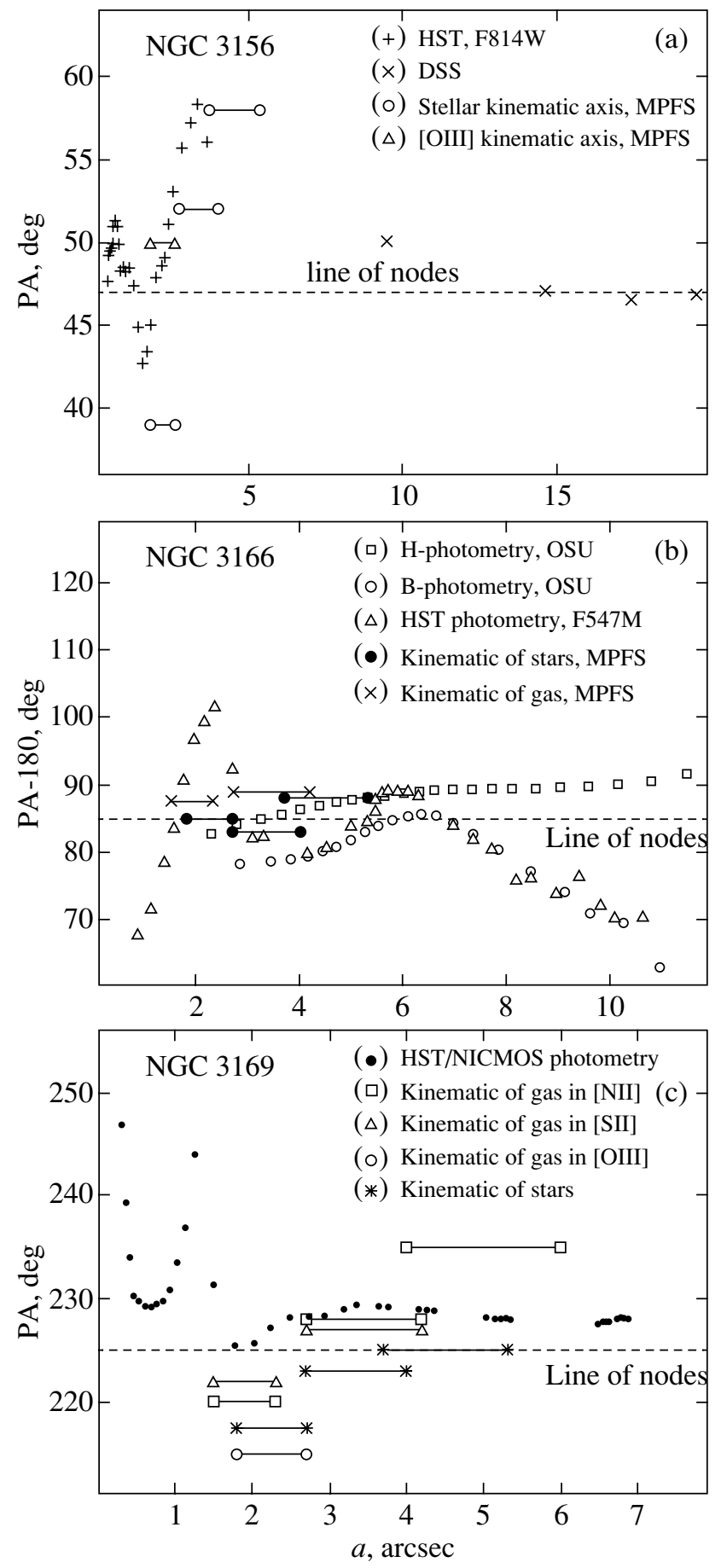

Fig. 6. Comparison of the orientations of the photometric and kinematic major axes for the three galaxies under study; the horizontal dotted line indicates the orientation of the line of nodes of the symmetry plane estimated from the outer isophotes belonging to the global stellar disks in the galaxies.

a kind of an anticyclone with respect to the global rotation of the group.

Finally, note another curious feature. We found a compact bar in the center of NGC 3169 (there is no global bar in the galaxy; see Eskridge et al. 2002). At the same time, the radial distribution of the stellar population age shows a characteristic ring structure: the nucleus and the $6^{\prime \prime}-7^{\prime \prime}$ ring zone have the youngest age. We have already encountered such a geometry of circumnuclear star formation bursts in the lenticular galaxies NGC 4429 and NGC 7013 (Sil'chenko and Afanasiev 2002); in the above paper, we associated these rings of younger stars with resonance structures in a triaxial potential: both observations and theory are known to place the so-called nuclear rings of star formation at the inner Lindblad resonance of bars. We seem to have found the same phenomenon in NGC 3169-a ring circumnuclear star formation burst triggered 1 Gyr ago by a concentration of gas flowing to the center near the inner Lindblad resonance of a central bar.

\section{ACKNOWLEDGMENTS}

We wish to thank A.V. Moiseev and V.V. Vlasyuk for support of the MPFS observations on the 6-m telescope. We used observational data obtained with the Jacobus Kapteyn telescope on the La Palma island operated by the Royal Greenwich Observatory at the Spanish del Roque de los Muchachos Observatory of the Institute of Astrophysics of the Canary Islands and taken from the publicly accessible archive of the Isaac Newton Group at the Astronomical Data Center, Great Britain. We also used data from the NASA/ESA Hubble Space Telescope operated by the Association of Universities for Research in Astronomy under contract with NASA, NAS 526555. During our work, we relied on the capabilities of the Lyon-Meudon Extragalactic Database (LEDA) provided by the LEDA team at the Lyon CRAL Observatory (France) and the NASA/IPAC database (NED) operated by the Jet Propulsion Laboratory of the California Institute of Technology under contract with the National Aeronautics and Space Administration (United States). This work was supported by the Russian Foundation for Basic Research, project no. 04-02-16087.

\section{REFERENCES}

1. V. L. Afanasiev, S. N. Dodonov, S. V. Drabek, and V. V. Vlasyuk, MPFS: User Manual (SAO RAN, Nizhnii Arkhyz, 1996) [in Russian].

2. V. L. Afanasiev, S. N. Dodonov, and A. V. Moiseev, Stellar Dynamics: from Classic to Modern, Ed. by L. P. Osipkov and I. I. Nikiforov (St. Petersburg, 2001), p. 103.

3. R. Bacon, G. Adam, A. Baranne, et al., Astron. Astrophys., Suppl. Ser. 113, 347 (1995). 
4. I. S. Balinskaya and O. K. Sil'chenko, Preprint No. 90, SAO RAN (Special Astrophysical Observatory, Russ. Acad. Sci., 1992).

5. A. Burgess, Mon. Not. R. Astron. Soc. 118, 477 (1958).

6. P. B. Eskridge, J. A. Frogel, R. W. Pogge, et al., Astrophys. J., Suppl. Ser. 143, 73 (2002).

7. G. Gilmore and R. F. G. Wyse, Astrophys. J. 367, L55 (1991).

8. M. P. Haynes, Astron. J. 86, 1126 (1981).

9. L. C. Ho, A. V. Filippenko, and W. L. Sargent, Astrophys. J., Suppl. Ser. 112, 315 (1997).

10. I. D. Karachentsev, Astron. J. 129, 178 (2005).

11. A. Mahdavi, H. Bohringer, M. J. Geller, and M. Ramella, Astrophys. J. 534, 114 (2000).

12. G. Marconi, F. Matteucci, and M. Tosi, Mon. Not. R. Astron. Soc. 270, 35 (1994).

13. F. Matteucci, Astron. Astrophys. 288, 57 (1994).

14. A. V. Moiseev and V. V. Mustsevoi, Pis'ma Astron. Zh. 26, 657 (2000) [Astron. Lett. 26, 565 (2000)].

15. J. S. Mulchaey, D. S. Davis, R. F. Mushotsky, and D. Burstein, Astrophys. J., Suppl. Ser. 145, 39 (2003).

16. S. E. Schneider, Astrophys. J. 288, L33 (1985).

17. S. E. Schneider, Astrophys. J. 343, 94 (1989).

18. O. K. Sil'chenko, Pis'ma Astron. Zh. 19, 693 (1993) [Astron. Lett. 19, 279 (1993)].
19. O. K. Sil'chenko, Astron. Zh. 71, 7 (1994) [Astron. Rep. 38, 3(1994)].

20. O. K. Sil'chenko, Astrophys. J. 641, 229 (2006).

21. O. K. Sil'chenko and V. L. Afanasiev, Astron. Astrophys. 385,1 (2002).

22. O. K. Sil'chenko, A. V. Moiseev, V. L. Afanasiev, et al., Astrophys. J. 591, 185 (2003).

23. G. Stasinska and I. Sodré, Jr., Astron. Astrophys. 374, 919 (2001).

24. D. Thomas, C. Maraston, and R. Bender, Mon. Not. R. Astron. Soc. 339, 897 (2003).

25. J. L. Tonry, A. Dressler, J. P. Blakeslee, et al., Astrophys. J. 546, 681 (2001).

26. S. C. Trager, S. M. Faber, G. Worthey, and J. J. Gonzàlez, Astron. J. 119, 1645 (2000).

27. H. D. Tran, Z. Tsvetanov, H. C. Ford, et al., Astron. J. 121, $2928(2001)$.

28. V. V. Vlasyuk, Astrofiz. Issled. (Izv. SAO RAN) 36, 107 (1993).

29. G. Worthey, Astrophys. J., Suppl. Ser. 95, 107 (1994).

30. G. Worthey, S. M. Faber, J. J. Gonzalez, and D. Burstein, Astrophys. J., Suppl. Ser. 94, 687 (1994).

31. A. V. Zasov and O. K. Sil'chenko, Astron. Zh. 60, 1063 (1983) [Sov. Astron. 27, 616 (1983)].

Translated by V. Astakhov 